\title{
Photometry and Spectrum measurements of the Laser Guide Star beam emission at Observatorio del Roque de Los Muchachos with the Gran Telescopio CANARIAS
}

\author{
G. Lombardi ${ }^{* a, b, c}$, D. Bonaccini Calia ${ }^{\mathrm{d}}$, M. Centrone ${ }^{\mathrm{e}}$, M. Reyes Garcia Talavera ${ }^{\mathrm{b}}$, T.J. Morris ${ }^{\mathrm{f}}, \mathrm{T}$. \\ Pfrommer $^{\mathrm{d}}$, A.G. Basden ${ }^{\mathrm{f}}$, F. Vidal ${ }^{\mathrm{g}}$, E. Gendron ${ }^{\mathrm{g}}$, G. Rousset ${ }^{\mathrm{g}}$, R.M. Mayers ${ }^{\mathrm{f}}$, J. Osborn ${ }^{\mathrm{f}}$, A.P.

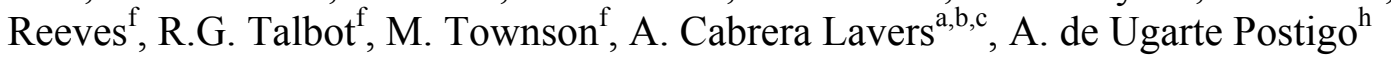 \\ ${ }^{a}$ Gran Telescopio CANARIAS S.A., c/ Cuesta de San José s/n, E-38712 Breña Baja, La Palma, Spain; \\ ${ }^{b}$ Instituto de Astrofísica de Canarias, E-38205 La Laguna, Tenerife, Spain; \\ 'Departamento de Astrofísica, Universidad de La Laguna, E-38206 La Laguna, Tenerife, Spain; \\ ${ }^{\mathrm{d}}$ European Southern Observatory, Karl-Schwarzschild-Str. 2, D-85748 Garching bei Muenchen, Germany; \\ ${ }^{\mathrm{e}}$ INAF-OAR National Institute for Astrophysics, Via Frascati 33, I-00078 Monte Porzio Catone (RM), Italy; \\ ${ }^{\mathrm{f}}$ Department of Physics, Durham University, South Road, Durham DH1 3LE, UK; \\ ${ }^{g}$ LESIA, Observatoire de Paris CNRS, Place Jules Janssen, F-92190 Meudon, France; \\ ${ }^{\text {h}}$ Instituto de Astrofísica de Andalucía, CSIC, Glorieta de la Astronomía s/n, E-8008 Granada, Spain
}

\begin{abstract}
In the past years ESO has built together with industry a transportable Laser Guide Star Unit (WLGSU) to test the laser technology while supporting some strategic experiments for the LGS-AO technologies. Since July 2016, the WLGSU has been installed at the Observatorio del Roque de Los Muchachos (ORM) in La Palma, next to the William Herschel Telescope (WHT), to perform different field experiments and studies related to the LGS technologies for Adaptive Optics. To study the photometric and spectral contamination from the scattering near the laser beam along the uplink at different heights, including the LGS itself, four observing runs, each of four hours, have been granted under Program GTC3-16ITP using the OSIRIS spectro-imager at the $10.4 \mathrm{~m}$ Gran Telescopio CANARIAS (GTC). We report preliminary results of the first two runs on the photometric and spectroscopic measurements and on the observed Raman emission of atmospheric molecules along the uplink beam.
\end{abstract}

Keywords: Laser Guide Star, Adaptive Optics, Laser Tomography Adaptive Optics, Atmospheric Characterization

\section{INTRODUCTION}

ESO has built together with industry a transportable Laser Guide Star (LGS) Unit, i.e. a compact system, in which an experimental 20W CW laser at $589 \mathrm{~nm}$ based on the ESO patented Fiber Raman Amplifier technology, is attached directly to a $30 \mathrm{~cm}$ launch telescope mounted on high precision pointing motors ([1]). The so-called "Wendelstein Laser Guide Star Unit" (WLGSU) has been designed, assembled, tested in the ESO laboratory and finally commissioned successfully in the summer of 2011, in Bavaria, Germany ([2]). The goal of the WLGSU is to field test the laser technology while supporting some strategic experiments for the LGS-AO technologies.

The use of a 589nm laser for LGS at the Canary Islands is ongoing since January 2015, when the ESO Wendelstain Laser Guide Star Unit has been installed in Tenerife at the Observatorio del Teide to carry on return flux experiments ([3], [4], [5], [6], [7]). Since July 2016, the WLGSU has been installed at the Observatorio del Roque de Los Muchachos (ORM) in La Palma, next to the William Herschel Telescope (WHT), to perform different field experiments and studies related to the LGS technologies for Adaptive Optics ([8]).

The photometry and the emission spectrum measured on the uplink beam are important for the operations aspects of the astronomical observatories with several telescopes on site. Software tools are used to avoid that a telescope at the observatory crosses the laser uplink beam while pointing and tracking a science target.

*gianluca.lombardi@gtc.iac.es; phone 34922425 720; gtc.iac.es 

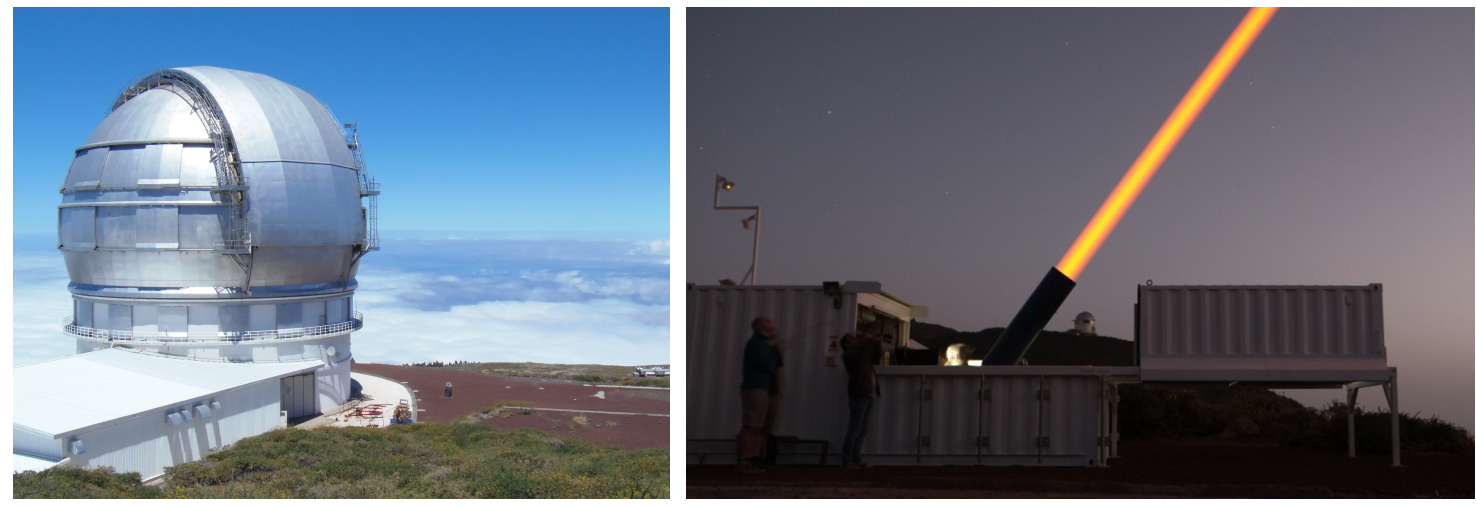

Figure 1. (left) the Gran Telescopio CANARIAS; (right) the ESO Wendelstain Laser Guide Star Unit at ORM.

Furthermore, it is important to avoid that photometric and spectral contamination from the scattering in the vicinity of the laser beam disturbs the observations. At which distance from the laser beam should the software prevent observations/laser propagation? The measurement of the scattering from the uplink beam is thus needed.

In order to investigate the emission spectrum and the photometry along the uplink laser beam at different heights, including the LGS itself, four observing runs, each of four hours, have been granted under Program GTC3-16ITP using the OSIRIS spectro-imager at the $10.4 \mathrm{~m}$ Gran Telescopio CANARIAS (GTC). We report preliminary results of the first two runs, performed on 17 and 18 September 2016, on the photometric and spectroscopic measurements and on the observed Raman emission of atmospheric molecules along the uplink beam.

\section{SETUP AND OBSERVING STRATEGY}

OSIRIS is a spectro-imager for the optical wavelength range, located in the Nasmith B focus of GTC. It provides standard Broad Band imaging and Long Slit Spectroscopy, and additional capability such as the Narrow Band Tunable Filters imaging, charge-shuffling and Multi Object Spectroscopy ([9], [10]). OSIRIS covers the wavelength range from 0.365 to $1.05 \mu \mathrm{m}$ with a total field of view of $7.8 \times 8.5 \operatorname{arcmin}(7.8 \times 7.8$ arcmin unvignetted), and $7.5 \times 6.0$ arcmin, for direct imaging and MOS respectively.

For this experiment, we have used OSIRIS with two instrument modes:

1. IMAGING: to obtain the photometry of the laser uplink in order to measure the contamination introduced by the beam scattering.

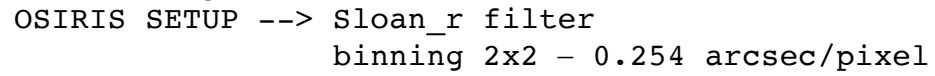

2. SPECTROSCOPY: to investigate the spectrum emission from the laser uplink beam.

OSIRIS SETUP - > R2500R + GR + slit0.6 arcsec

binning $1 \times 1-0.508 \AA /$ pixel

Figure 2 shows the relative positions of the GTC and the WLGSU at the Observatorio del Roque de Los Muchachos, while Table 1 reports the coordinates for both. The WLGSU and the GTC are about $1113 \mathrm{~m}$ away in SW-NE direction, with a difference in elevation (telescope pupil) of about $31 \mathrm{~m}$ (Figure 3).

The observations have been performed under clear or photometric conditions. We have pointed the GTC telescope toward the laser plume and the uplink beam at different heights (Figure 3). Because of the strong parallax due to the distance between the GTC and the laser launch telescope, parallax-corrected pointing coordinates for the GTC have been calculated using a Python script. We could intercept both the plume and the uplink beam at the desired height $100 \%$ of the time.

After every pointing, the laser mount and the telescope tracking have been stopped in order to acquire the data and avoid the rotation due to the parallax. A strong defocus of the GTC M2 has been necessary in order to focus the laser plume and the uplink. 
Table 1. Coordinates of the GTC and the WLGSU at ORM.

\begin{tabular}{|c|c|c|c|}
\hline & Longitude & Latitude & Elevation \\
\hline GTC & $17^{\circ} 53^{\prime} 30^{\prime \prime} .8 \mathrm{~W}$ & $28^{\circ} 45^{\prime} 23^{\prime \prime} .8 \mathrm{~N}$ & 2300 m.a.s.I. \\
\hline WLGSU & $17^{\circ} 52^{\prime} 54^{\prime \prime} .1 \mathrm{~W}$ & $28^{\circ} 45^{\prime} 38^{\prime \prime} .1 \mathrm{~N}$ & 2331 m.a.s.I. \\
\hline
\end{tabular}

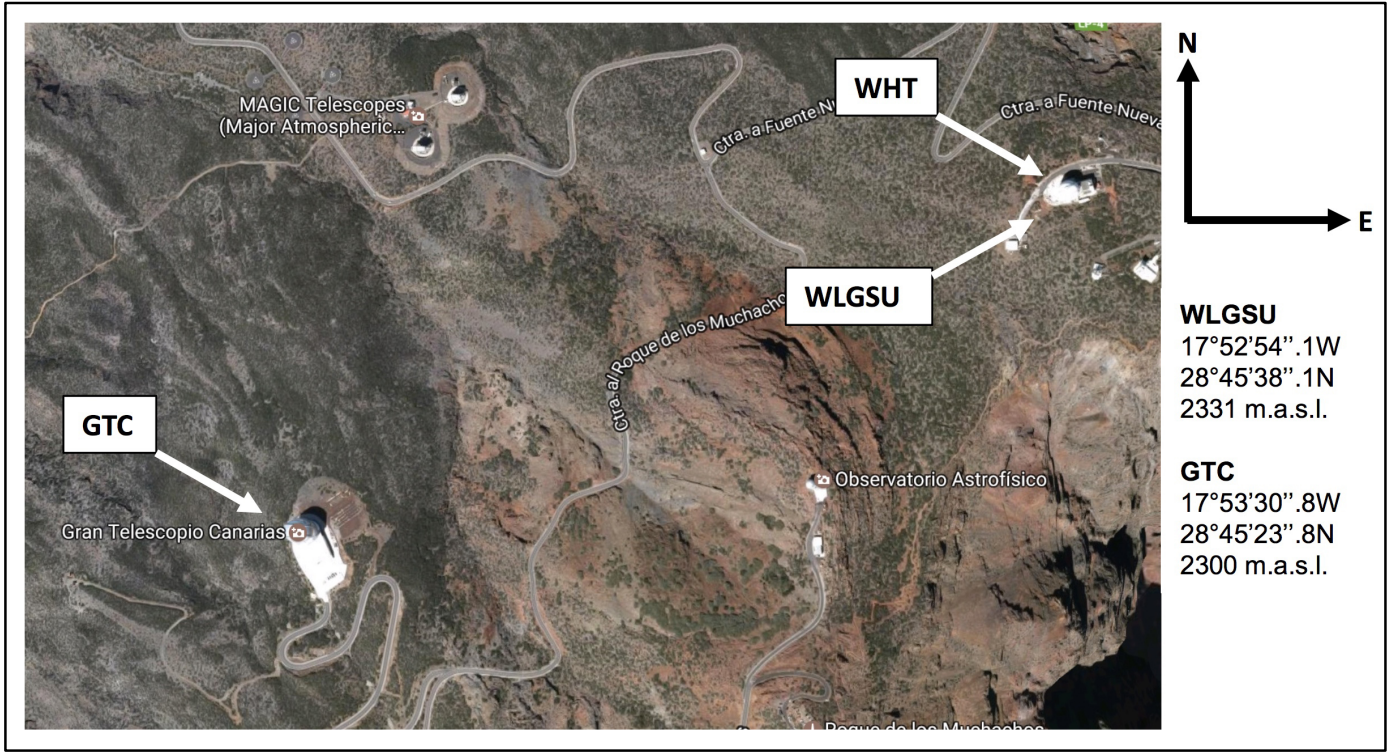

Figure 2. Relative positions of the GTC and the WLGSU at ORM.

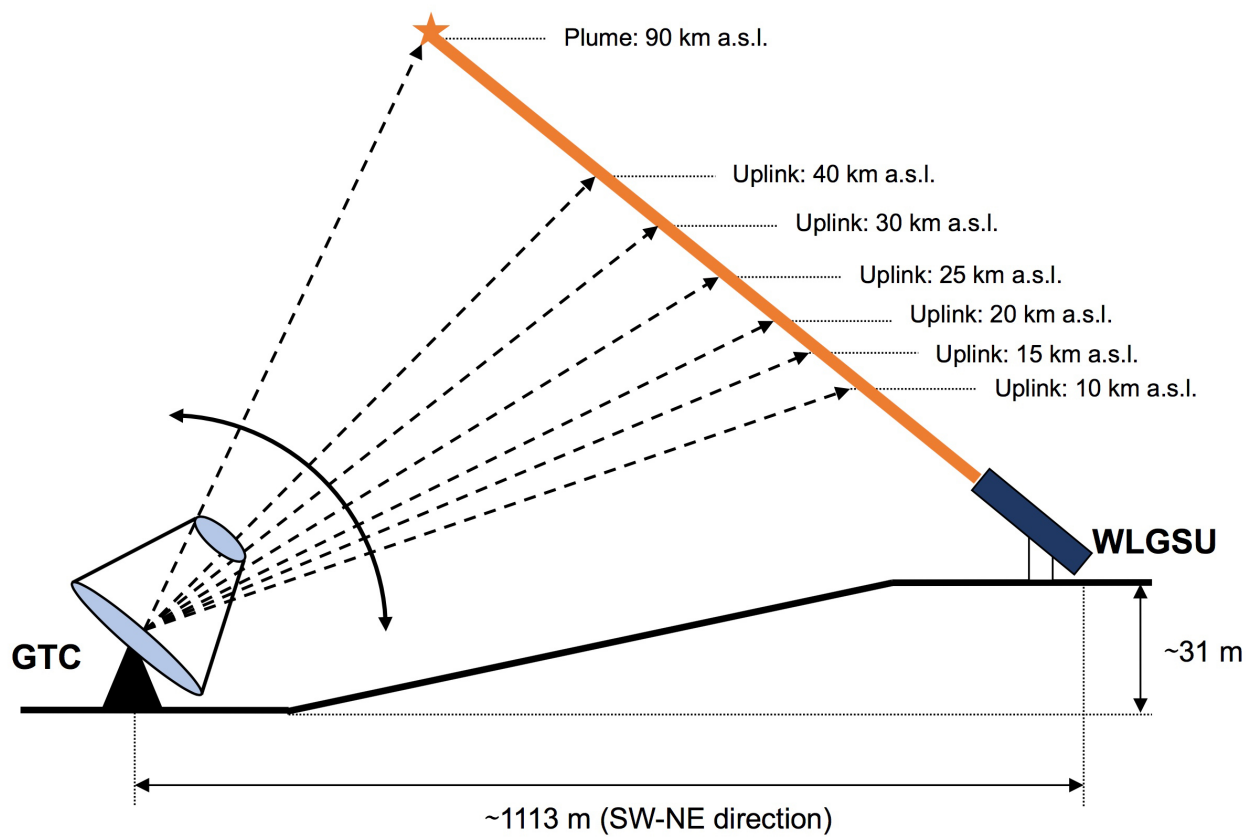

Figure 3. Schematic representation of the strategy used for the observations of the laser uplink beam and the LGS with the GTC. 

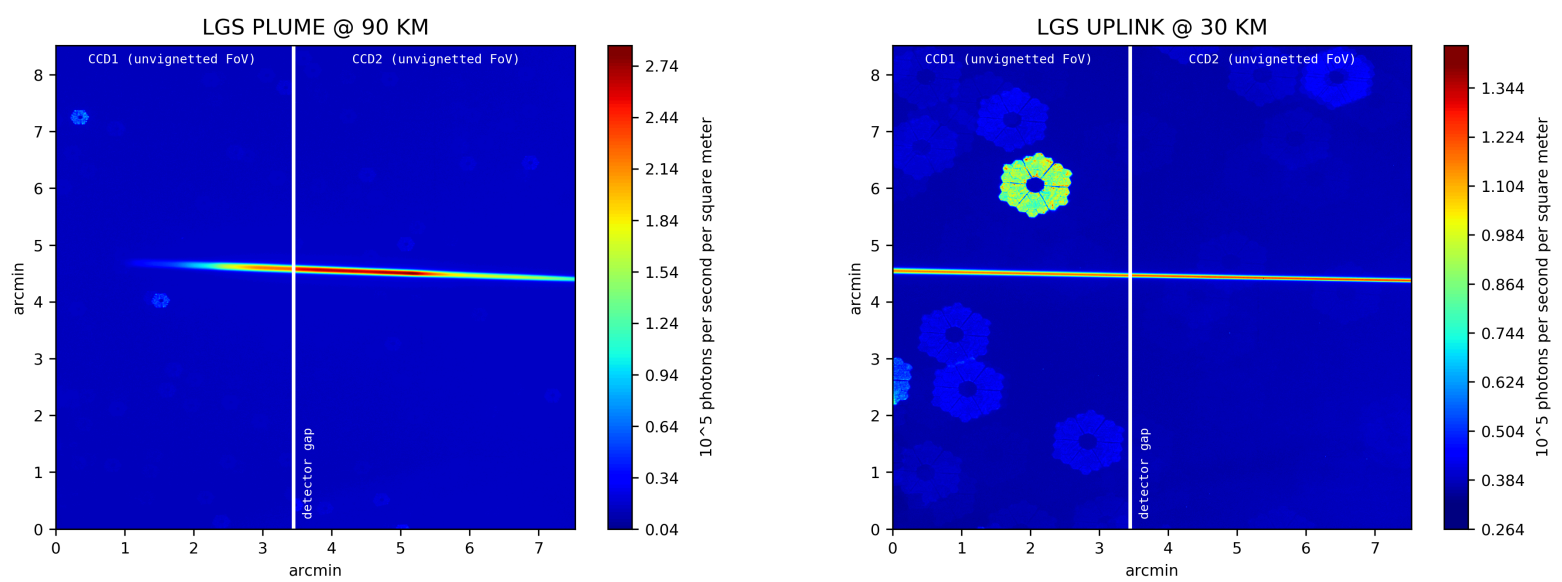

Figure 4. Fluxes for the LGS plume and the uplink beam at $30 \mathrm{~km}$. The original OSIRIS calibrated images have been cut, so that the presented figures correspond to the unvignetted Field of View for both detector CCDs

\section{PHOTOMETRY}

For data reduction, OSIRIS Sloan_r images have been debiased, flat-fielded and corrected for distortion. The Photometric Standard SA95-193 has been acquired in the same nights and used for flux calibration. It is important to note that for these first two run, we have not acquired the sky with laser OFF, so no sky subtraction has been applied to the reduced images.

The atmospheric extinction for a wavelength of $589 \mathrm{~nm}$ at ORM is 0.09 mag per airmass ([11]). The measured OSIRIS magnitude for SA95-193 is 14.9795, while we expect to find 13.9491 at the ground at the star airmass. The above gives an instrument extinction of $1.03 \mathrm{mag}$, that correspond to an overall throughput of $39 \%$.

The OSIRIS detector QE is $78 \%$ around $(589 \pm 200) \mathrm{nm}$, while the Sloan_r filter transmissivity at $589 \mathrm{~nm}$ is $90.4 \%$, furthermore the GTC has an effective collecting area of $73 \mathrm{~m}^{2}$. We have converted the SA95-193 total ADU counts into the flux in photons per second per square meter. We have scaled the fluxes taking into account the difference in spectral behavior of the system between the Sloan $\mathrm{r}$ central wavelength $(658 \mathrm{~nm})$ and $589 \mathrm{~nm}$.

Figure 4 show the fluxes for the LGS plume and the uplink beam at $30 \mathrm{~km}$. The original OSIRIS calibrated images have been cut, so that the presented figures correspond to the unvignetted Field of View for both detector CCDs.

It is important to note that these are partial and preliminary results, since the 3rd and 4th runs of this experiment will be performed in July 2017. Furthermore, no sky subtraction has been applied to these results.

From these first results it looks that the scattering of the uplink beam might be considered negligible in an area of less than 0.5 arcmin for the beam, while for the LGS plume, the scattering extends up to about 0.5 arcmin around the plume.

\section{SPECTROSCOPY}

We have investigated the spectrum emission from the laser uplink beam at different heights. Data have been acquired using the R2500R grism and a slit of 0.6 arcsec. The acquisition procedure is as for the Photometry case and for the same reasons, calculating the parallax-corrected coordinates and stopping the telescope tracking after every pointing.

The OSIRIS instrument rotator has been positioned to have the slit parallel to the beam, so that the beam was superposed all along the slit (see Figure 5). For every height, we have acquired the spectra with the laser ON and OFF to retrieve the sky and subtract it from the uplink spectra in order to isolate the laser emission lines. The spectra have been fluxcalibrated using the Spectrophotometric Standard g191-B2B observed during the same night. 


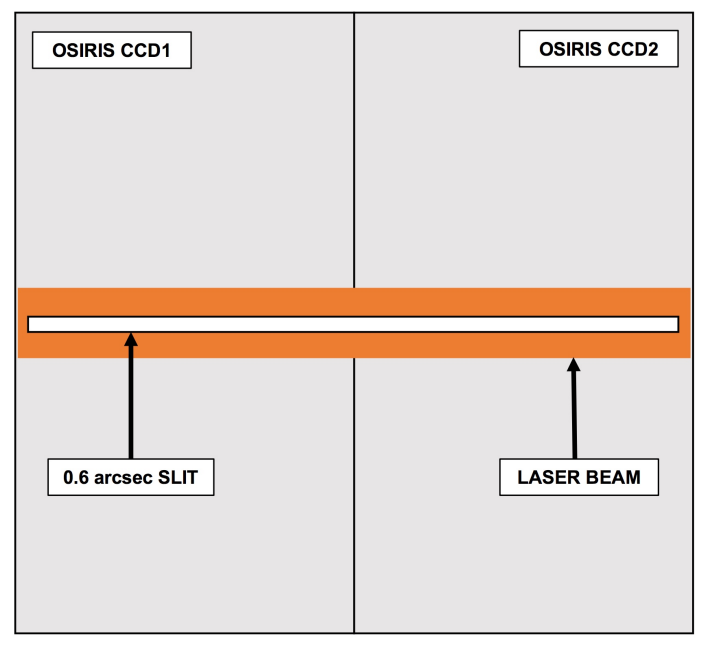

Figure 5. Schematic representation: for the Spectroscopy observations, the OSIRIS instrument rotator has been positioned to have the slit parallel to the beam, so that the beam was superposed all along the slit.

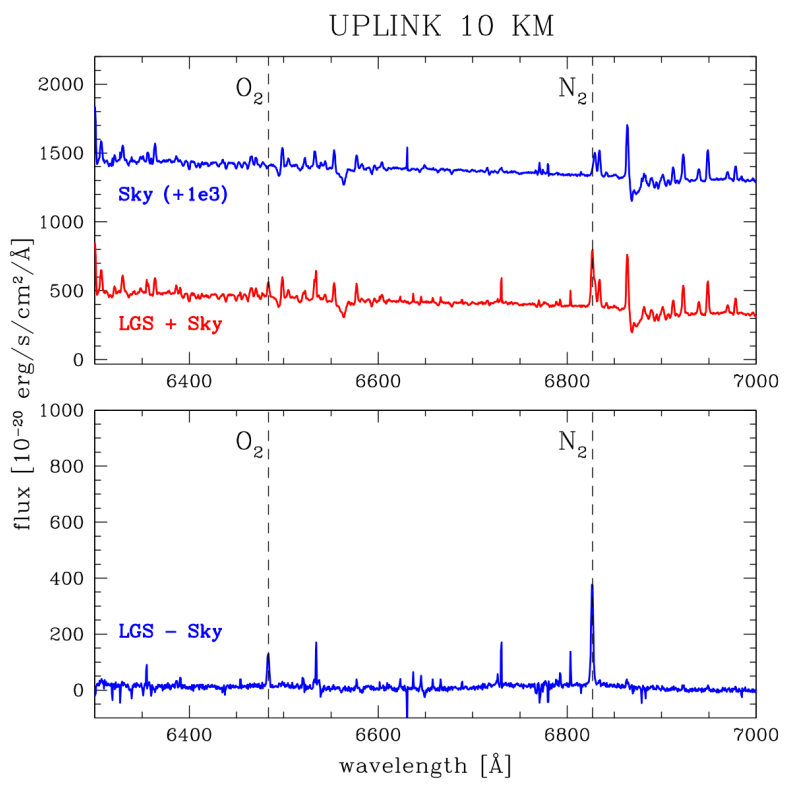

Figure 6. Spectra of the sky and the uplink at $10 \mathrm{~km}$ as well as the laser emission after sky subtraction. Raman emissions for $\mathrm{O}_{2}$ and $\mathrm{N}_{2}$ are clearly visible. Some spurious emissions from a star passing through the slit during the exposure must be ignored.

Figure 6 shows an example of spectra of the sky and the uplink at $10 \mathrm{~km}$ as well as the laser emission after sky subtraction. Raman emissions for $\mathrm{O}_{2}$ and $\mathrm{N}_{2}$ are clearly visible. Some spurious emissions from a star passing through the slit during the exposure must be ignored.

Figure 7 show the detail of the $\mathrm{O}_{2}$ and $\mathrm{N}_{2}$ Raman emissions for every height. The peak of the emission is at $25 \mathrm{~km}$, and disappear above, while the situation is unclear for the $\mathrm{O}_{2}$ emission at $20 \mathrm{~km}$ (a start passing through the slit?). The integrated fluxes are reported in Table 2.

It is important to note that these are partial and preliminary results, since the 3rd and 4th runs of this experiment have been performed in July 2017 and data have not been analyzed yet. In any case, it is interesting to report that similar results with the VLT at Paranal Observatory (at $25 \mathrm{~km}$ only) have been reported by Vogt et al. ([12]). 
Table 2. Integrated flux for LGS, $\mathrm{O}_{2}$ and $\mathrm{N}_{2}$ lines.

\begin{tabular}{|c|c|c|c|}
\hline \multirow[b]{2}{*}{ Height (a.s.I.) } & \multicolumn{3}{|c|}{ Integrated Flux $\left[10^{-20} \mathrm{erg} / \mathrm{s} / \mathrm{cm}^{2} / \mathrm{W}\right]$} \\
\hline & $10 \mathrm{~km}$ & $15 \mathrm{~km}$ & $20 \mathrm{~km}$ \\
\hline LGS (5889.96 Å) & $2.1 \times 10^{6}$ & $1.9 \times 10^{6}$ & $1.5 \times 10^{6}$ \\
\hline $\mathrm{O}_{2}(6484.39 \AA)$ & $3.2 \times 10^{1}$ & $2.7 \times 10^{1}$ & $\ldots$ \\
\hline $\mathrm{N}_{2}(6827.17 \AA)$ & $9.8 \times 10^{1}$ & $8.9 \times 10^{1}$ & $5.5 \times 10^{1}$ \\
\hline
\end{tabular}

\begin{tabular}{|c|c|c|c|}
\hline Height (a.s.I.) & $\mathbf{2 5} \mathbf{~ k m}$ & $\mathbf{3 0 ~} \mathbf{~ k m}$ & $\mathbf{4 0 ~} \mathbf{~ k m}$ \\
\hline LGS (5889.96 $\AA$ ) & $2.7 \times 10^{6}$ & $0.7 \times 10^{6}$ & $0.5 \times 10^{6}$ \\
\hline $\mathbf{O}_{\mathbf{2}}(\mathbf{6 4 8 4 . 3 9} \AA)$ & $3.9 \times 10^{1}$ & $1.0 \times 10^{1}$ & $<1.0 \times 10^{1}$ \\
\hline $\mathbf{N}_{\mathbf{2}}(\mathbf{6 8 2 7 . 1 7} \AA)$ & $1.2 \times 10^{2}$ & $2.6 \times 10^{1}$ & $2.4 \times 10^{1}$ \\
\hline
\end{tabular}
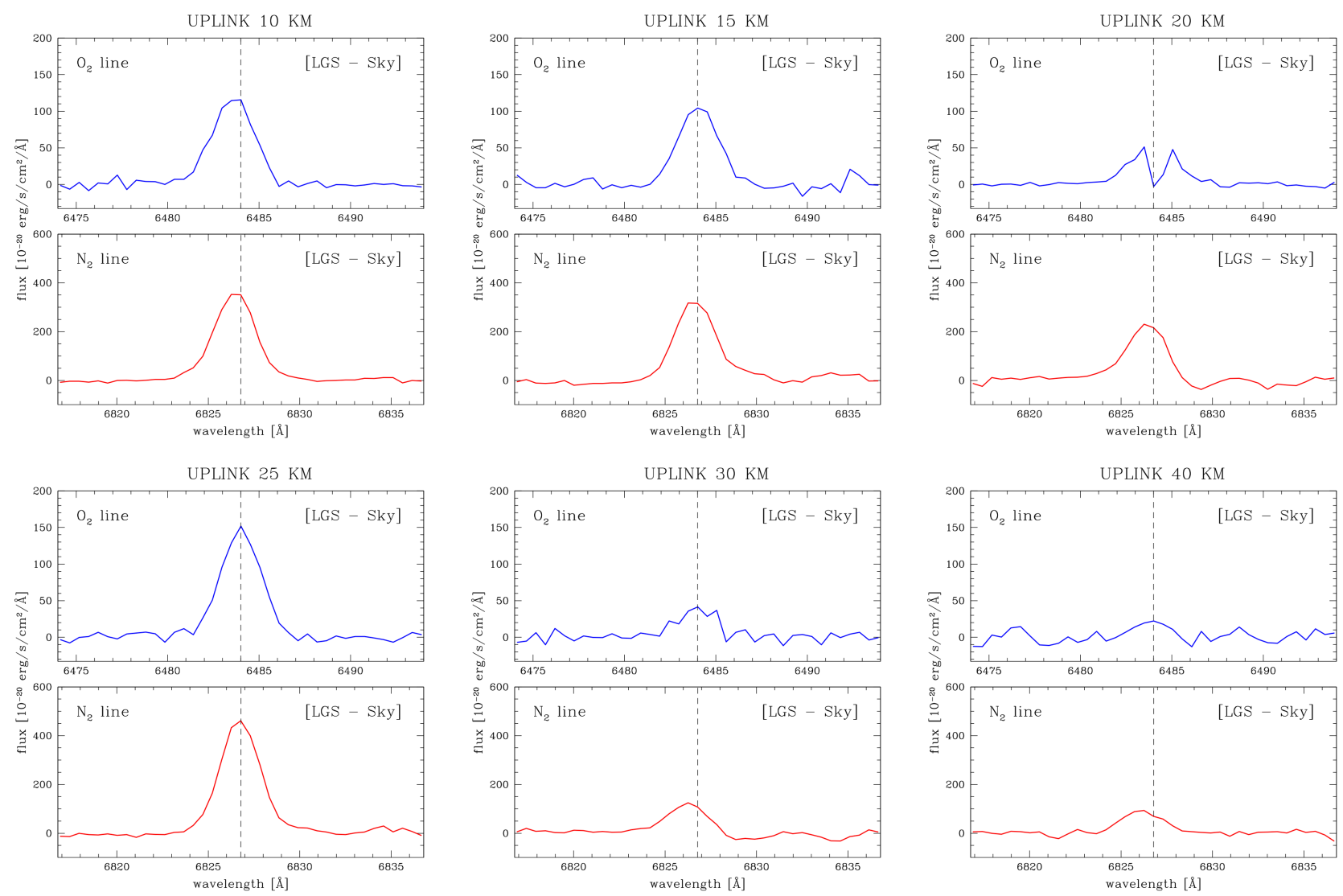

Figure 7. $\mathrm{O}_{2}$ and $\mathrm{N}_{2}$ Raman emissions for every height. The peak of the emission is at $25 \mathrm{~km}$, and disappear above, while the situation is unclear for the $\mathrm{O}_{2}$ emission at $20 \mathrm{~km}$ (a start passing through the slit?). 


\section{REFERENCES}

[1] Bonaccini Calia D., Friedenauer A., Protopopov V, Guidolin I, Taylor L.R., Karpov V., Hager M., Clements W., Ernstberger B., Lewis S., Kaenders W., "PM fiber lasers at $589 \mathrm{~nm}$ : a $20 \mathrm{~W}$ transportable laser system for LGS return flux studies", Proceedings of the SPIE, 7736, Adaptive Optics Systems II, 77361U, 2010

[2] Bonaccini Calia D., Holzlöhner R., Guidolin I, Pedichini F., Centrone M., Kasper T., Lombardi G., Forth W., Lewis S., Pfrommer T., Hackenberg W., "ELT LGS-AO: Optimizing the LGS Return Flux", Second AO4ELT Conference Adaptive Optics for Extremely Large telescopes, Victoria BC, 2011

[3] Bonaccini Calia D., Guidolin I., Friedenauer A., Hager M., Karpov V., Pfrommer T., Holzlöhner R., Lewis S., Hackenberg W., Lombardi G. et al., "The ESO Transportable LGS Unit for Measurements of the LGS Photon Return and Other Experiments", Proceedings of the SPIE, 8450, Modern Technologies in Space- and Ground-Based Telescopes and Instrumentation II, 84501R, 2012

[4] Holzlöhner R., Bonaccini Calia D., Bello D., Budker D., Centrone M., Guidolin I., Hackenberg W., Lewis S., Lombardi G., Montilla I., Pedichini F., Pfrommer T., Reyes Garcia Talavera M., Rochester S., "Comparison between Observation and Simulation of Return Flux with a 20W CW Laser on Tenerife", 10th Annual Workshop on Laser Technology and Systems for Astronomical Adaptive Optics, Lake Arrowhead CA, 2015

[5] Bonaccini Calia D., Guidolin I., Pfrommer T., Holzlöhner R., Lewis S., Hackenberg W., Lombardi G., Bello D., Centrone M., Pedichini F., Reyes Garcia Talavera M., Montilla I., "LGS field experiments at Observatorio de el Teide", 11th Annual Workshop on Laser Technology and Systems for Astronomical Adaptive Optics, Durham (UK), 2016

[6] Holzlöhner R., Bonaccini Calia D., Bello D., Budker D., Centrone M., Guidolin I., Hackenberg W., Lewis S., Lombardi G., Montilla I., Pedichini F., Pedreros Bustos F., Pfrommer T., Reyes Garcia Talavera M., Rochester S., "Comparison between observation and simulation of sodium LGS return flux with a 20W CW laser on Tenerife", Proceedings of the SPIE, 9909, Adaptive Optics Systems V, 99095E, 2016

[7] Holzlöhner R., Bonaccini Calia D., Guidolin I., Pfrommer T., Lewis S., Hackenberg W., Bello D., Budker D., Lombardi G., Bello D., Centrone M., Pedichini F., Reyes Garcia Talavera M., Montilla I., Rochester S., Budker D., "Comparison between Observation and Simulation of Return Flux with a $20 \mathrm{~W}$ CW Laser on Tenerife", 11th Annual Workshop on Laser Technology and Systems for Astronomical Adaptive Optics, Durham (UK), 2016

[8] Rousset G., Myers R., Bonaccini Calia D., Gratadour D., Morris T., Gendron E., Basden A., Buey T., Chemla F., Lombardi G., Osborn J., Pfroemmer T., Reeves A., Reyes M., Talbot G., Townson M., Vidal F., "Progress report of the ELT-elongated LGS on-sky experiment with WLGSU and CANARY at WHT", Fourth AO4ELT Conference Adaptive Optics for Extremely Large telescopes, Paper 107, Lake Arrowhead CA, 2015

[9] Cepa J, "OSIRIS: Final characterization and scientific capabilities", Astrophysics and Space Science Proceedings, 14:15, 2010

[10] Cepa J., Aguiar M., Escalera V.G., et al., "OSIRIS tunable imager and spectrograph", Proceedings of the SPIE, 4008, Optical and IR Telescope Instrumentation and Detectors, 623-631, 2000

[11] ING internal report: http://www.ing.iac.es/Astronomy/observing/manuals/ps/tech notes/tn031.pdf

[12] Vogt F., Bonaccini Calia D., Hackenberg W., Opitom C., et al., "Detection and Implications of Laser-Induced Raman Scattering at Astronomical Observatories", Physical Review X, 7, 021044, 2017 